\title{
Tapes für Wearables
}

\author{
Sie sind kleine Wunderwerke und gehören zu den jüngsten \\ technologischen Errungenschaften: Wearables. Die Minicomputer \\ werden direkt am Körper getragen und erledigen smarte Dienste \\ wie das Messen von Kalorienverbrauch, Bewegungsradius \\ und Schlafdauer.In ihrem Gehäuse befinden sich zahlreiche \\ hochempfindliche Elektronik-Bauteile, die miteinander verklebt \\ werden.
}

\section{Gunnar von der Geest}

Unter Experten gilt der Wearables-Markt als „The Next Big Thing“ innerhalb der Technologie-Branche. Nach Analysen der internationalen Wirtschaftsprüfungsgesellschaft PricewaterhouseCoopers (PwC) hatte der weltweite Markt für Wearables im Jahr 2015 einen Wert von 6,3 Milliarden Euro und wird bis 2018 um jährlich 21 Prozent wachsen. Der deutsche Branchenverband „Bitkom“ erwartet einen jährlichen Anstieg von 24 Prozent für den europäischen Markt, der damit im Jahr 2018 bis auf 8,8 Milliarden Euro klettern soll; die PwC-Prognose gibt mit einem durchschnittlichen jährlichen Wachstum von 25,2 Prozent bis 2018 einen noch optimistischeren Ausblick.Für Deutschland wird ein ähnlicher Zuwachs angenommen. Beliebteste Artikel sollen nach derzeitigem Erkenntnisstand die Smartwatches und Fitness-Armbänder bleiben.

\section{Enge Kooperation mit Global Playern}

„Auf diese beiden Kategorien haben wir uns auch fokussiert, als Tesa vor etwa drei Jahren zusammen mit Global Playern aus der Elektronikindustrie die Entwicklung neuartiger Klebebänder in Angriff nahm“, sagt Dr. Ronald Pfaff, Marketing Director der Business Unit Electronics. „Durch die frühzeitige und enge Kooperation sind wir nun in der Lage, OEMs und Zulieferern spezielle Tapes anzubieten, die den besonderen Anforderungen an das Kleben von Bauteilen in Wearables gerecht werden“, erläutert Pfaff. Gegenwärtig umfasst das Sortiment vier doppelseitige Klebebänder in den Dicken zwi- schen 50 und 250 Mikrometern. Anfang 2017 fanden bei potenziellen Kunden diverse Spezifizierungen für die in der Reinraum-Einheit des Tesa-Werkes Hamburg hergestellten Produkte statt.

\section{Tapes mit „Anti-Körpern“}

Da Wearables ständig bewegt werden, müssen die Tapes eine hohe Klebfestigkeit und Resistenz gegen Stöße aufweisen. Darüber hinaus sind die Geräte durch den engen Körperkontakt unterschiedlichen biologischen und chemischen Substanzen ausgesetzt. Hierzu gehören neben Schweiß auch Parfüm, Sonnencreme, Seife oder andere Flüssigkeiten beziehungsweise Gase. Wenngleich die Klebebänder nicht direkt auf der Haut aufliegen, so können doch Partikel der teilweise aggressiven Stoffe durch winzige Spalte ins Innere der Wearables eindringen und im schlimmsten Fall die Verklebung lösen. Aus diesem Grund verfügt das neue Quartett der doppelseitigen Tesa High Performance Tapes über entsprechende „AntiKörper“ gegen Chemie \& Co.

\section{Kleben von Deckglas und Gehäuse}

Bezüglich der Anwendungsbereiche konzentriert sich das Unternehmen bei Smartwatches vor allem auf die Verklebung von Deckglas (Lens Mounting) und Gehäuse (Back Cover Mounting). Darüber hinaus spielt insbesondere bei Fitness-Armbändern die sichere Fixierung der sensiblen Sensorik zum Tracken unterschiedlicher Körperfunktionen eine besondere Rolle.

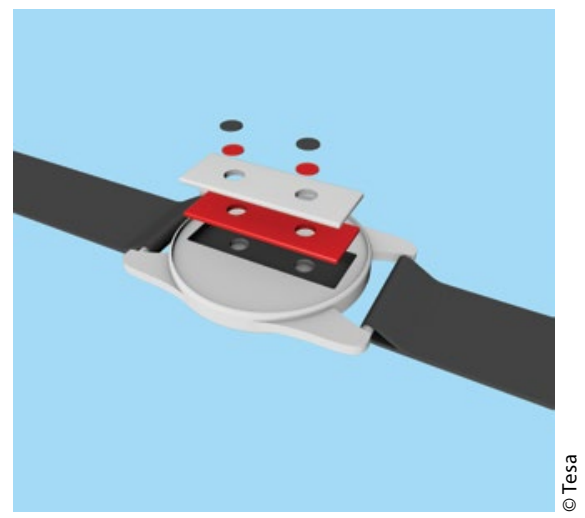

Bild 1 > Sensor Mounting: Im Gehäuse einer Smartwatch befinden sich zahlreiche hochempfindliche Elektronik-Bauteile, die miteinander verklebt werden. Die unterschiedlich dicken High-Tech-Tapes fertigt Tesa im Werk Hamburg-Hausbruch.

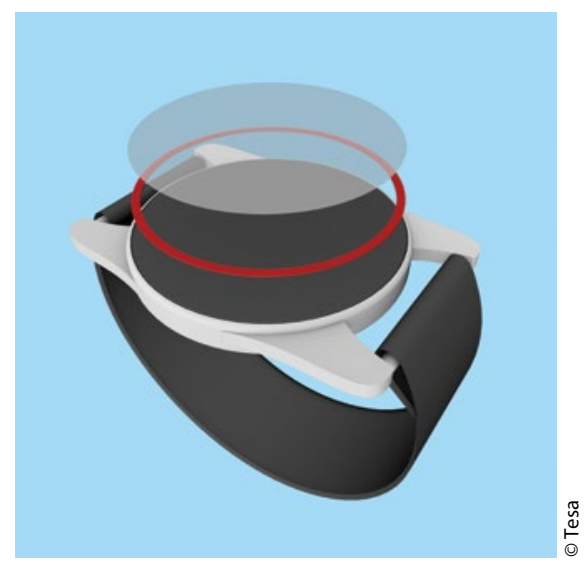

Bild 2 > Lens Mounting: Das Deckglas einer Smartwatch wird mit höchster Präzision auf dem Gehäuse fixiert. Die Anforderungen an das Tape (rot) sind vielfältig: Zum einen eine starke Verklebungsfestigkeit und Belastbarkeit bei Stößen - zum anderen eine Resistenz gegen chemische Substanzen.

Doch auch Daten-Brillen und mit Elektronik versehene Kleidung bieten im $\mathrm{Zu}$ kunftsmarkt der „Vermessung des Ich“ noch reichlich „Kleb-Stoff“.//

\section{Der Autor}

\section{Gunnar von der Geest}

(Gunnar.vonderGeest@tesa.com) ist als Manager Corporate Communications bei der Tesa SE tätig. Er verantwortet unter anderem den Bereich Fach- und Wissenschaftsmedien für die Business Unit Tesa Industry. 